\title{
The Function of Roots of Tea Plant (Camellia sinensis) Cultured by a Novel Form of Hydroponics and Soil Acidification
}

\author{
Kieko Saito $^{1^{*}}$, Masahiko Ikeda ${ }^{2}$ \\ ${ }^{1}$ Institute for Environmental Sciences, University of Shizuoka, Shizuoka, Japan; ${ }^{2}$ College of Environment and Disaster Research, Fuji \\ Tokoha University, Fuji, Japan. \\ Email: ${ }^{*}$ saitok@u-shizuoka-ken.ac.jp
}

Received February $2^{\text {nd }}, 2012$; revised March $1^{\text {st }}, 2012$; accepted March $20^{\text {th }}, 2012$

\begin{abstract}
A novel form of hydroponic culture was employed to explore the physiological function of roots of a tea plant (Camellia sinensis). The $\mathrm{pH}$ of the nutrient solution with an actively growing tea plant decreased during cultivation. Furthermore, no oxalic acid, tartaric acid, malic acid or citric acid, all possible factors in acidification, was detected in the nutrient solution of a growing plant. A proton pump inhibitor suppressed the acidification of the solution. Soil acidification might have been accelerated with a proton released from ammoniacal nitrogen preferentially for the growth, suggesting the specific mechanism of tea plant as a functional food.
\end{abstract}

Keywords: Acid Soil; Hydroponic Culture; Organic Acid; Tea; Root

\section{Introduction}

Plants use unique physiological functions and growth characteristics to protect themselves against natural enemies and environmental stress such as draught and chemicals. The roots of a plant play an important role in growth, and they are related to the biosynthesis that adds to the plant's leaves. It is well known that most of the plants release organic acids to acidify the soil, leading making it easier for plants to take easily metals, such as iron, into the body for the growth by solubilization; moreover, and most plants release organic acids from the root to combine with aluminum, a trace element in soil, to prevent the intake of aluminum, which is toxic for growth $[1,2]$. These mechanisms are important because it is impossible for plants to move like animals [3].

Morita et al. [4] reported that a tea plant (Camellia sinensis) absorbed aluminum from the roots and then combined it with oxalic acid inside its body in order to inhibit the aluminum's toxicity. A tea leaf contains catechins, GABA ( $\gamma$-aminobutyric acid), and teanine as secondary metabolites, which have functions in the human body when tea is consumed as a beverage. In addition, a tea plant exhibits remarkable growth under acidic conditions and aluminum supply, which are its specific properties [5].

Recent excessive use of fertilizers containing nitrate

"Corresponding author. has caused acidification of the soil around tea fields, leading to environmental pollution, and this has become a serious problem. However, it could not be determined with certainty whether the roots would release the organic acids needed to naturally acidify the soil as done by most plants, even though an acidic soil is suitable for the growth of tea plants, because soil cultivation has disturbed the conditions for investigating the specific role of roots in detail.

This is the first study to explore the physiological function of the roots of a tea plant cultivated by a novel form of hydroponics to examine the effect of the roots zone.

\section{Materials and Methods}

For the samples used in this study, tea cuttings (var. Yabukita) were cultivated in a soil for several months until rooting, and then rooted tea cuttings were transplanted to a nutrient solution ( $\mathrm{pH} 4.3$ ) containing aluminum $(10.8 \mathrm{mg} / \mathrm{L})$ based on Hoagland's solution [6]. The plant was cultured in the solution with continuous aeration under controlled conditions in an incubator (Biotron, Nihonika, Japan). Day/night temperatures were kept at $25 / 18^{\circ} \mathrm{C}$, photosynthetic photon flux density (PPFD) at the plant was $40.0 \mu \mathrm{mol} \cdot \mathrm{m}^{-2} \cdot \mathrm{s}^{-1}$ during a $12 \mathrm{~h}$ daytime, and the relative humidity was about $60 \%$. The $\mathrm{pH}$ in the nutrient solution was measured at room temperature us- 
ing a $\mathrm{pH}$ meter (Horiba, Japan). The organic acids of the nutrient solution were analyzed using a HPLC system (Shimadz, LC10AD, LC-10AD pump, a SPD-10AV UVVis detector controlled via Class LC-10 data acquisition software, Japan) equipped with a column (Showa Denko K. K, Shodex RSpac KC-811, Japan) [7]. Organic acid peaks (oxalic acid, tartaric acid, malic acid and citric acid) were identified by comparing their UV-Vis spectral characteristics and retention times with those from commercial standards supplied by Wako Pure Chemicals Industry, Ltd., Japan. $\mathrm{NH}_{4}^{+}-\mathrm{N}$ in the solution was measured by an indophenol blue absorptiometric method [8], and Erythrosine B (Wako Pure Chemicals Industry, Ltd., Japan) was employed as a proton pump inhibitor.

\section{Results and Discussion}

The change in $\mathrm{pH}$ in the nutrient solution was measured for nine days. The $\mathrm{pH}$ of the solution in a plant with vital roots gradually decreased from $\mathrm{pH} 4.3$ to 3.8 . However, no change was seen in the solution of a damaged plant without vital roots, which was damaged with an alkaline solution for seven days before the experiment (data not shown). Moreover, a high correlation was seen between the consumption of the solution and the $\mathrm{pH}$ decrease during growth (Figure 1). There was no effect of evaporation of the nutrient solution on $\mathrm{pH}$ change, while having many roots did contribute to a quick decrease in the $\mathrm{pH}$ (data not shown). The change in $\mathrm{pH}$ in the solution might be an indicator of a tea plant's growth; in other words, the acidification of a sphere of roots is obviously the distinctive function of a tea plant's growing process, indicating that specific substance(s) released from the roots possibly cause the decrease in $\mathrm{pH}$.

It was reported that citric and malic acids are secreted from roots for strong tolerance to aluminum under the ground $[3,9]$. Organic acids were thought to be key sub-

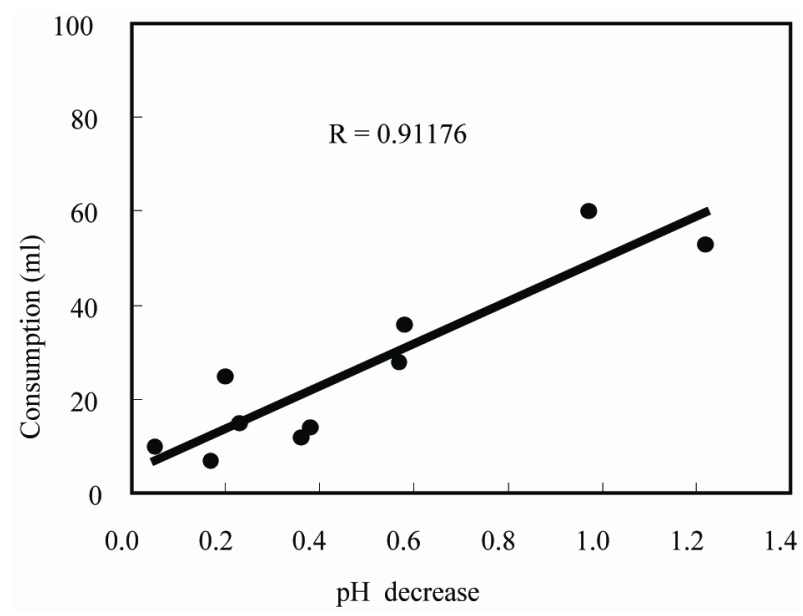

Figure 1. Consumption of nutrient solution and $\mathrm{pH}$ decrease in the growth culture. stances that acidify the nutrient solution in which a tea plant is cultivated; however, oxalic, tartaric, malic and citric acids, which are the main organic acids of plants, were not detected in this study's analysis of a solution of active tea plants after the cultivation. Consequently, this observation shows that the roots of a tea plant did not release organic acid(s) to acidify the solution (Table 1).

The $\mathrm{pH}$ of a solution is basically a quantitative disequilibrium of the cation and anion, and thus the acidification would be caused by the positive action of roots toward growth, which is a possible function. Most ions absorbed by the root are nitrogenous ions $\left(\mathrm{NH}_{4}^{+}-\mathrm{N}\right.$ and $\mathrm{NO}_{3}^{-}-\mathrm{N}$ ) as nutrition, but ammonium and nitrate ions exist in the nutrient solution as well as in soil. That is, roots absorb ammonium ion, which leads to acidification of the soil, while absorption of nitrate ions leads to alkalinity. The concentration of $\mathrm{NH}_{4}^{+}-\mathrm{N}$ in the solution was measured during growth, and the results showed that $\mathrm{pH}$ decreased with $\mathrm{NH}_{4}^{+}-\mathrm{N}$ consumption, since there was a high correlation between these activities (Figure 2). This result shows that the acidification of the nutrient solution was probably caused by proton release from the roots due to the absorption of ammonium. In Figure 3, a proton pump inhibitor, Erythrosine B, which specifically reacts with the protoplasmatic membrane, was used to confirm the effect of a proton on the $\mathrm{pH}$ decrease in the solution [3]. The proton pump inhibitor suppressed the acidification of the solution in a dose-dependent manner, indicat-

Table 1. Analysys of organic acids in the nutrient solution.

\begin{tabular}{lcccc}
\hline \multirow{2}{*}{ Orgaic acid } & \multicolumn{4}{c}{ Incubation time (day) } \\
\cline { 2 - 5 } & 1 & 4 & 7 & 10 \\
\hline L-Malic acid & ND & ND & ND & ND \\
Tartaric acid & ND & ND & ND & ND \\
Oxalic acid & ND & ND & ND & ND \\
Citric acid & ND & ND & ND & ND \\
\hline
\end{tabular}

ND: not detected.

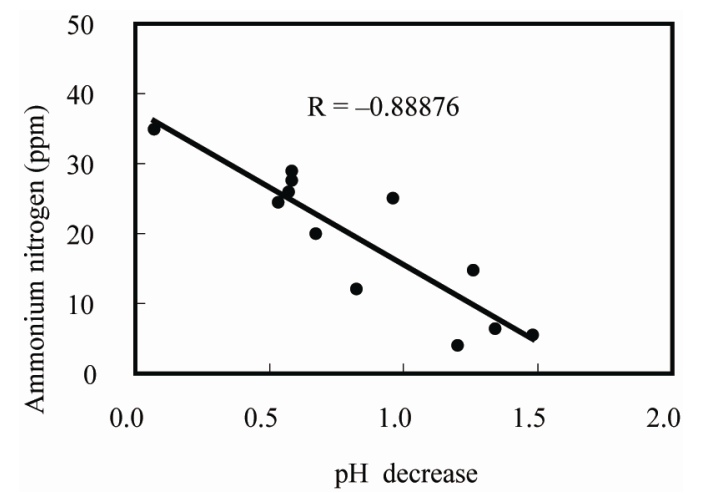

Figure 2. Ammoniacal nitrogen consumption and $\mathrm{pH}$ change in the nutrient solution. 


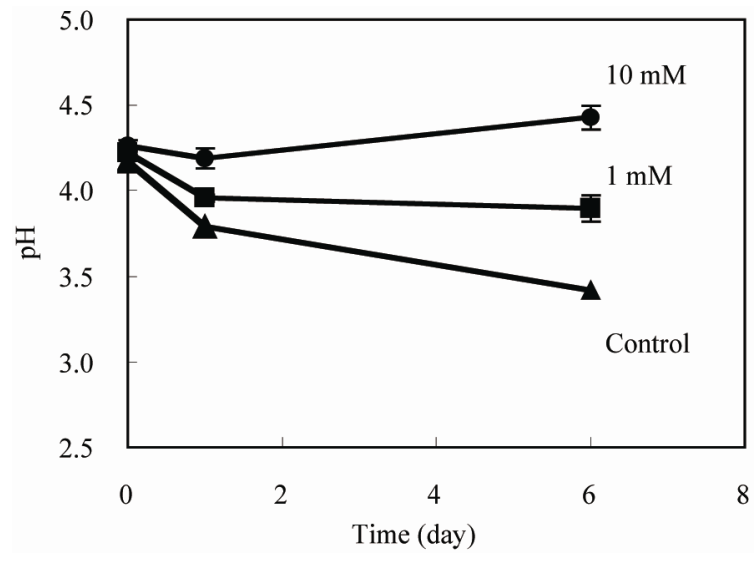

Figure 3. Effect of proton pump inhibitor on the acidification of nutrient solution. $\bullet: 10$ mM Erythrosine B, a: $1 \mathrm{mM}$ Erythrosine B, $\boldsymbol{\Delta}$ : control.

ing that proton from ammonium ion obviously decreased $\mathrm{pH}$ (Figure 3). These results were supported by a report of Dr. Konishi [5] that a tea plant likes $\mathrm{NH}_{4}^{+}-\mathrm{N}$ much more than $\mathrm{NO}_{3}^{-}-\mathrm{N}$ as nitrogenous nutrition. This property of roots may lead to produce functional metabolites for human health. Especially theanine, an amino acid, synthesized in the roots, cannot be found in any other plant except for one non edible mushroom.

In conclusion, the acidification of soil was probably accelerated by a proton released from $\mathrm{NH}_{4}^{+}-\mathrm{N}$ in the course of nutrient adsorption for growth, and may related with the functional metabolites. The novel form of hydroponics revealed the effect of the function of a tea plant's roots on soil, and the findings could contribute to new possibility for physiological function of a tea as a functional food other than a solution to the environmental pollution of acidic soil around tea fields.

\section{Acknowledgements}

The authors are grateful to Dr. Konishi (emeritus professor of Shizuoka University) for his valuable advice and to Mr. Miyamoto for his assistance (The Herb Garden of
University of Shizuoka).

\section{REFERENCES}

[1] L. V. Kochian, "Cellular Mechanisms of Aluminum Toxicity and Resistance in Plants," Annual Review of Plant Physiology and Plant Molecular Biology, Vol. 46, 1995, pp. 237-260. doi:10.1146/annurev.pp.46.060195.001321

[2] H. Matsumoto, "Cell Biology of Aluminum Toxicity and Tolerance in Higher Plants," International Review of $\mathrm{Cy}$ tology, Vol. 200, 2000, pp. 1-46. doi:10.1016/S0074-7696(00)00001-2

[3] A. Higa, E. Miyamoto and Y. Kitamura, "Root Tip-Dependent, Active Riboflavin Secretion by Hyoscyamus albus Hairy Roots under Iron Deficiency," Plant Physiology and Biochemistry, Vol. 46, No. 4, 2008, pp. 452-460. doi:10.1016/i.plaphy.2008.01.004

[4] A. Morita, O. Yanagisawa, S. Takatsu, S. Maeda and S. Hiradate, "Mechanism for the Detoxification of Aluminum in Roots of Tea Plant (Camellia sinensis (L.) Kuntze)," Phytochemstry, Vol. 69, No. 1, 2008, pp. 147153. doi:10.1016/j.phytochem.2007.06.007

[5] S. Konishi, S. Miyamoto and T. Taki, "Stimulatory Effects of Aluminum on Tea Plants Grown under Low and High Phosphorus Supply," Soil Science and Plant Nutrition, Vol. 31, 1985, pp. 361-368.

[6] S. Konishi, "Promotive Effects of Aluminum on Tea Plant Growth," Japan Agricultural Research (JARQ), Vol. 26, 1992, pp. 26-33.

[7] Y. Hernández, M. G. Lobo and M. González, "Factors Affecting Sample Extraction in the Liquid Chromatographic Determination of Organic Acids in Papaya and Pineapple," Food Chemistry, Vol. 114, No. 2, 2009, pp. 734-741. doi:10.1016/i.foodchem.2008.10.021

[8] Japanese Industrial Standard JIS K-0102, "The Indophenol Blue Absorptiometric Method," Japanese Standards Association, Vol. 42, 1991.

[9] J. F. Ma, P. R. Ryan and E. Delhaize, “Aluminium Tolerance in Plants and the Complexing Role of Organic Acids," Trends in Plant Science, Vol. 6, No. 6, 2001, pp. 273-278. doi:10.1016/S1360-1385(01)01961-6 